\title{
Introducing electron capture into the unitary-convolution-approximation energy-loss theory at low velocities
}

\author{
G. Schiwietz ${ }^{1, *}$ and P. L. Grande ${ }^{2}$ \\ ${ }^{1}$ Helmholtz-Zentrum Berlin für Materialien und Energie GmbH, Institute G-I2, Hahn-Meitner-Platz 1, DE-14109 Berlin, Germany \\ ${ }^{2}$ Instituto de Física, Universidade Federal do Rio Grande do Sul, BR-91500-970 Porto Alegre, Brazil
}

(Received 1 September 2011; published 3 November 2011)

\begin{abstract}
Recent developments in the theoretical treatment of electronic energy losses of bare and screened ions in gases are presented. Specifically, the unitary-convolution-approximation (UCA) stopping-power model has proven its strengths for the determination of nonequilibrium effects for light as well as heavy projectiles at intermediate to high projectile velocities. The focus of this contribution will be on the UCA and its extension to specific projectile energies far below $100 \mathrm{keV} / \mathrm{u}$, by considering electron-capture contributions at charge-equilibrium conditions.
\end{abstract}

DOI: 10.1103/PhysRevA.84.052703

PACS number(s): 34.50.Bw, 34.70.+e, 34.10.+x, 03.65.Sq

\section{INTRODUCTION}

Theoretical investigations of the energy losses of charged particles interacting with gases and solids started about a century ago. These early investigations on the interaction of fast light ions with matter mainly focused on ionization processes. In the remaining part of this paper, we will use the term "fast projectiles" to indicate projectile speeds beyond the orbital velocities of most weakly bound electrons (say, $E_{p} / \mathrm{M}_{p} \gg 1 \mathrm{MeV} / \mathrm{u}$ ), a case where many electron shells contribute to the ion energy loss. With "slow projectiles" we mean that the projectile interacts nearly exclusively with only the valence or conduction band, at incident velocities clearly below the corresponding Fermi velocity (say, $E_{p} / \mathrm{M}_{p} \ll$ $20 \mathrm{keV} / \mathrm{u}$ ). In this work, we try to consider all important electronic energy-loss processes [1].

Figure 1 shows that ionization is in fact the main energyloss component at high projectile speeds $v_{p}$. We may see that even at high energies, excitation processes may not be neglected, if an accurate energy-loss description is envisaged. At low speeds, all reaction processes can be important, and specifically target and projectile excitation may constitute a strong or even the main energy-loss component. Ionization is often of minor importance for slow atomic or ionic projectiles, but may dominate for antiprotons or negative muons [2].

Electron capture is indicated in Fig. 1 by a shaded area bordered by two curves that are separate by orders of magnitude at low speeds. The transfer of target electrons to the bound projectile state (electron capture) leads to a so-called capture-and-loss cycle, where the captured electron is lost by the projectile in a subsequent collision, involving a projectile energy loss as well. The influence of electron capture depends strongly on the projectile charge state and, specifically at low velocities, on matching conditions for the electronic energy levels of the target and the projectile (resonant or nonresonant electron capture) [3]. The electroncapture cross section is exactly zero for antiprotons (because of the negative projectile charge), it is small for asymmetrical systems such as protons on helium (due to nonresonant capture processes), but resonant electron capture (specifically for

\footnotetext{
*schiwietz(at)helmholtz-berlin.de
}

homonuclear systems such as $\mathrm{H}^{+}+\mathrm{H}$ ) involves enormous total cross sections. The resulting strong variation of the stopping power due to electron transfer (electron capture) dependent on the collision system is probably the reason for conflicting statements on the importance of capture. Uncertainties of the (extended) Firsov model might be one of the reasons why some models suggest that the influence of electron capture at low velocities is enormous [4], while Sigmund [5] and others estimate that charge exchange yields only a minor contribution.

This paper is focused on recent developments of the unitary convolution approximation (UCA) and on partial ion energyloss cross sections for specific projectile charge states and energy-transfer processes, extending the range of validity to much lower projectile energies. We present theoretical CASP energy-loss results in comparison to experimental data. We will show that electron-capture processes may not be neglected for gas targets, neither for light nor for heavy target systems.

Most current energy-loss models are based on the impactparameter method [6]. This method describes the nuclear motion using classical trajectories (often straight-line trajectories) and it is highly accurate for most electronic processes above a few $\mathrm{keV}$. It is noted that all energy-loss models known to us are based on the independent-electron picture, if localized electron states are considered (with the exception of Ref. [7]). The only current energy-loss theory that involves no further approximations is the atomic-orbital coupled-channel (AOCC) theory. Note that we have applied this coupled-channel method successfully for the computation of energy losses, mainly for collisions of light ions with few-electron targets, in order to limit the computing time to reasonable values [8-11]. In this work, we have computed AOCC results as a benchmark for our more approximate UCA model, to be discussed further below.

There are various other approximate solutions $[2,12,13]$ of the energy-loss problem for bound target states at low velocities or for valence-band electrons [14]. Other methods cover somewhat larger energy ranges [15]. A simplified electron-gas model by Arista and Lifschitz, based on a generalization of the Friedel sum rule [16], has been extended from low to intermediate velocities and to heavier ions as well [17]. Purely classical descriptions of energy losses [18,19] may be used specifically for heavy ions, but this involves a variety of uncertainties: at high projectile speeds the dipole terms are suppressed, and at low speeds, quantum mechanical tunneling and 


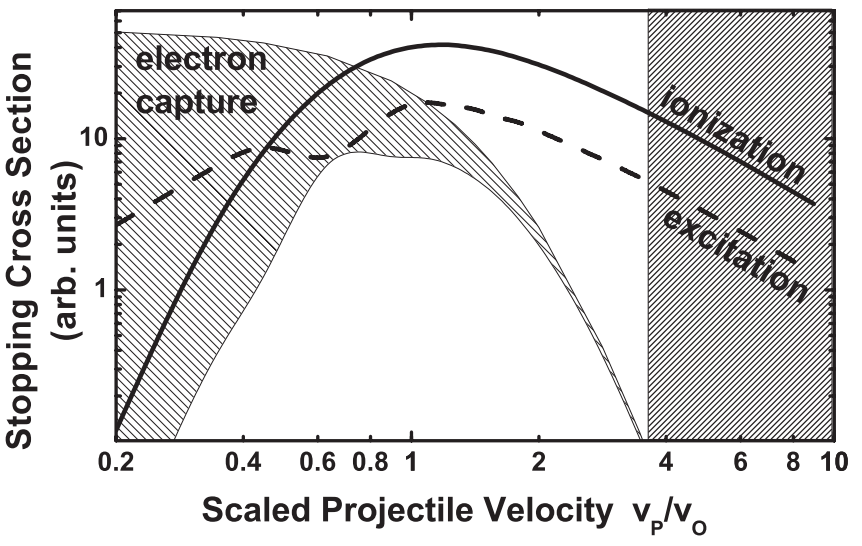

FIG. 1. Typical behavior of basic single-electron processes as a function of the reduced projectile velocity $v_{p} / v_{o}$ for a fixed projectile charge state $\left(v_{o}\right.$ is the mean orbital velocity of a certain specific state). The energy dependences of stopping cross sections related to excitation, ionization, and electron capture are shown schematically in the plot. The corresponding stopping cross sections have been extracted from experimental and theoretical cross sections and mean energy transfers for $\mathrm{H}$ and $\mathrm{He}$ targets (from Ref. [3]).

quasimolecular orbital effects and details of the electron scattering are of course also missing in pure classical treatments.

Some years ago, a very promising simplified model with its foundation at intermediate energies was proposed [20] as an extension of Bohr's classical stopping theory. This "binary theory" of electronic stopping has been extended and its use is suggested by the authors for extremely low up to relativistic speeds, excluding only the lightest projectile-target systems [5,21]. The target-electron binding is incorporated in the binary theory by a mapping to a projectile screening function that delivers the required suppression of low energy transfers (defined in the Bohr regime of stopping). In addition, this screening function also accounts for static and dynamic projectile screening and the target characteristics are furthermore determined by oscillator strengths. So-called shell corrections add to the improvement of the target description (the initial internal electron motion). Relativistic corrections are incorporated as well. This model accounts for the (mean) projectile charge and incorporates electron loss and projectile excitation. The so-called Barkas effect is included by a binary momentum-transfer treatment that is sensitive only to the short-range part of the screening function. Our analysis indicates that long-ranged polarization effects (an important part of the Barkas effect, e.g., for proton-rare-gas collisions) are not fully included in the binary theory, similar to the short-ranged binding effect being missing (and of course other quasimolecular orbital effects). This suggests that (apart form possible intrinsic uncertainties) this model might not be very accurate at low velocities.

At intermediate to high energies, the continuum-distortedwave eikonal-initial-state (CDWEIS) model may be applied for solving the time-dependent Schrödinger equation. CDWEIS goes far beyond perturbation theory and thus, the resulting ionization cross sections and stopping cross sections are very close to experimental data [22] or to AOCC results. However, it involves some non-negligible numerical efforts. The considerably simpler convergent kinetic Lindhard theory
(CKLT) model by Maynard et al. was initially devised for heavy ions in plasmas and has been extended to neutral target systems as well [23]. It is also applicable to intermediate and high energies.

Consideration of the projectile charge fractions, the socalled charge-state approach [15,24,25], is typical for modern energy-loss treatments. In principle, it should involve summation of all energy losses from capture and target ionization plus excitation for each charge state $q$ with the contribution due to loss and excitation of projectile electrons, resulting in the total stopping power, respectively, stopping cross section, $S_{e}$, for the collision system in question. Specifically at high ion velocities, theory often relies on first-order perturbation theory for excitation and ionization, also named first-order semiclassical approximation [6,26] in the impact-parameter picture or equivalently, first Born approximation or Bethe theory (not to be confused with the simplified Bethe formula, the asymptotic high-energy solution) [27].

\section{THE UCA MODEL AND CASP PROGRAM}

The program CASP makes use of the convolution approximation [either the perturbative convolution approximation (PCA) [28,29] or the more advanced UCA [30,31]]. The physical inputs of the program [32] are the projectile velocity, the projectile-screening potential, the target-electron density distribution (which we have tabulated using results from our Hartree-Fock-Slater code [33,34]), and the oscillator strengths for the target electrons. The code is based on an exact matching of the quantum mechanical mean electronic energy transfers for the asymptotic regions of very small and very large impact parameters. The UCA is a model with very general applicability (not restricted to a certain range of impact parameters or projectile-target combinations). This model has undergone many test phases and has been continuously improved over the years. The corresponding CASP computer code (convolution approximation for swift projectiles) includes many different modes of operation and may freely be downloaded [32]. The UCA model includes a simple relativistic correction [35] (no radiation energy loss and no relativistic density effect) and thus, it becomes inaccurate only for kinetic energies per nucleon exceeding several hundred $\mathrm{MeV} / \mathrm{u}$.

The code is not restricted to Coulombic point charges as screening of the projectile charge is fully included [36]. This means that we average energy losses over the projectile charge-state fraction (including electron-loss processes) or (for heavy ions) one may simply estimate the energy loss for the (noninteger) mean charge. Although there is some knowledge on the charge-state evolution [37], only limited information (derived from $\mathrm{x}$ rays [38] and $\delta$ electrons [39]) exists on the excitation state of the projectile ions inside matter. As even the most advanced current theoretical charge-state treatments involve a somewhat limited accuracy and limited parameter ranges (somewhat below ten bound electrons are feasible nowadays [40]), accurate charge-state fits for ions in solids and also in gases [41] are still a necessary ingredient for accurate stopping-power calculations. Further corrections and ingredients extend the UCA range of validity towards lower energies and these improvements are mentioned in the following. 
At intermediate energies, the Barkas (polarization) effect $[42,43]$ (related to a modification of the target electron density via long-ranged dipole forces) is important for an accurate stopping-power prediction. Similar to the binding effect, these energy-loss contributions are also sign-of-charge dependent (related to odd powers of the projectile nuclear charge). At small impact parameters, however, the screening of the projectile nuclear charge will introduce another mechanism related to second-order perturbation theory, namely, a shortranged Barkas (screening) effect. The screening leads to a distance-dependent variation of the forces and correspondingly of the electron trajectory. The net effect is typically a significant energy-loss enhancement for positive projectile charges. This latter mechanism is included in the CASP program using an ansatz similar to the binary model by Sigmund and Schinner [5].

The PCA model is not an exact solution of first-order perturbation theory, mainly because it is based on a peaking approximation that erases any influence of momentum-space components. Thus, a corresponding correction, the so-called shell correction, has to be applied. In general, shell corrections account for intrinsic uncertainties in the description of the initial target states and usually also for deficiencies of the treatment of the electron dynamics. Such a highly accurate shell correction (shell-effect renormalization [44]) has been applied by scaling the PCA dynamics to exact first-order Born cross-section results for a hydrogenlike target model. In an intermediate step, we make use of the kinetic theory [45] or the binary model by Sigmund and Schinner [5] for consideration of the detailed Fourier-space distribution (computed from our numerical target wave functions) [44].

Another aspect that is improved in the current UCA version is the kinematical treatment of electron-loss processes at intermediate energies. Of course, projectile excitation is converted directly into a projectile energy loss. However, electron-loss processes (projectile ionization) at high-energy transfers involve only a minor interaction of the outgoing electron with the projectile core. For these cases, the projectile energy loss is strongly suppressed. We use the derivation [46] of Sigmund and Glazov for the kinematic suppression together with high precision AOCC computations, in order to derive the first accurate projectile energy-loss results of this type [44].

\section{SLOWING DOWN DUE TO ELECTRON CAPTURE PROCESSES}

As we have shown recently [44], there is another important energy-loss contribution in addition to excitation and ionization of target or projectile electrons, namely, electron capture (transfer of bound target electrons to the moving projectile-ion system). In that work, we have estimated the electron-capture contributions from experimental electroncapture cross sections. In the following, however, we will invoke purely theoretical estimates for the electron-capture contribution in the case of equilibrium stopping cross sections related to equilibrium projectile charge distributions. The electron-capture contribution $S_{e}^{\text {cap }}$ to the electronic stopping cross section is

$$
S_{e}^{\mathrm{cap}}=\sum_{q=1}^{Z_{p}} f(q) \sigma^{\mathrm{cap}}(q) \Delta E^{0}(q)
$$

where $q$ is the projectile charge state, $f(q)$ is the equilibrium charge-state distribution (obtained from fits [41]), $\Delta E^{\text {cap }}(q)$ is the mean energy transfer corresponding to an average over individual capture events for an initial charge state $q$, and $\sigma^{\text {cap }}(q)$ is the total electron-capture cross section involving all target shells at a fixed projectile charge. The energy transfer is estimated from the first (static) target ionization potential $I_{\mathrm{tar}, 1}$, the binding energy of the lowest unoccupied projectile state, which is equal to the first (static) ionization potential $I_{\text {proj; } 11}(q-1)$ of the projectile after the capture process, and the projectile velocity $v_{p}$ (all quantities are expressed in atomic units (a.u.), with $e=m_{e}=\hbar=1$ ):

$$
\Delta E^{\mathrm{cap}}(q)= \begin{cases}v_{p}^{2} / 2+\left[1-0.5 I_{\mathrm{proj} ; 1}(q-1) / I_{\mathrm{tar} ; 1}\right] \min \left(2 I_{\mathrm{tar} ; 1}, v_{p}^{2} / 2\right), & \text { for } \quad I_{\mathrm{proj} ; 1}(q-1)>I_{\mathrm{tar} ; 1} \\ v_{p}^{2} / 2+I_{\mathrm{tar}, 1}-I_{\mathrm{proj} ; 1}(q-1)+\min \left(2 I_{\mathrm{proj} ; 1}(q-1), v_{p}^{2} / 2\right) / 4, & \text { for } \quad I_{\mathrm{proj} ; 1}(q-1) \leqslant I_{\mathrm{tar}, 1}\end{cases}
$$

$\Delta E^{\text {cap }}$ [see further below for a detailed discussion of Eq. (2)] is written as a sum of (1) the translational energy $\operatorname{los} \Delta \Delta E_{\text {trans }}^{\text {cap }}=$ $v_{p}^{2} / 2$ [the first term of both lines in Eq. (2)] and (2) an estimated mean dynamic excitation energy $\Delta E_{\text {exc }}^{\text {cap }}$ [given by the last terms of both lines in Eq. (2)], which is the difference of mean electron-binding energies before and after the capture event.

Figure 2 is used to outline typical transitions in electroncapture events from the valence band to bound states of the moving projectile, corresponding to the mean dynamic excitation energy. Here we distinguish four cases (low and high projectile velocity as well as low and high projectile binding energy) as discussed in the following. It is emphasized that the acceleration of the active electron during the transfer from the target to the projectile system of reference (the translational energy-loss term $v_{p}^{2} / 2$ ) is not accounted for in any of the Figs. 2(a)-2(d). We make use of knowledge on electron capture in fast ion-atom collisions as described by the boundary-corrected first-order Born theory [47], by the equivalent true first-order Born approximation [48] theory or the eikonal theory [49], where capture is distributed nearly equally over excited projectile states up to the continuum limit (partial waves of $s$ type are favored). For slow projectiles the resonant energetic channel is strongly favored and high angular momentum states of the projectile are involved, as described, e.g., by the classical over-the-barrier model $[50,51]$.

This means for a slow projectile with a high ionization potential [see Fig. 2(a)] that capture will occur via resonant 
(a)

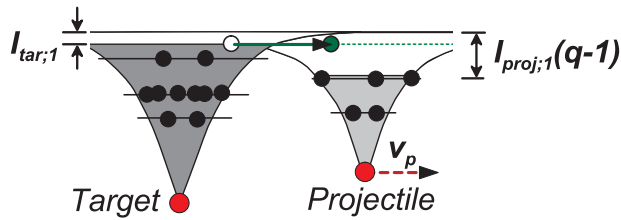

(b)

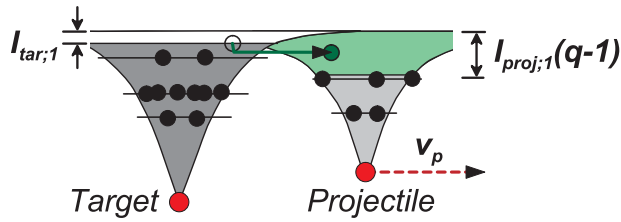

(c)

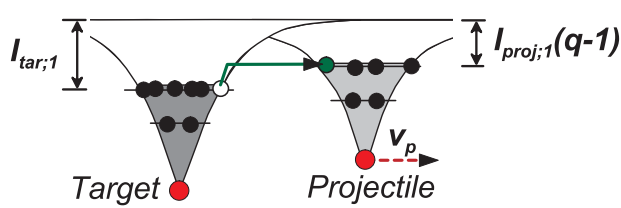

(d)

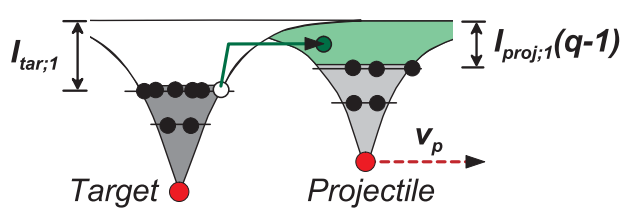

FIG. 2. (Color online) Bound-state energetics for electron capture from the valence-band states for different relative speeds and binding energies in the target and projectile systems. (a) and (c) refer to low projectile speeds $v_{p}$ and (b) and (d) refer to high speeds. (a) and (b) refer to low target binding energies (compared to the projectile) and (c) and (d) refer to high target binding energies.

or near-resonant channels, corresponding to $\Delta E_{\mathrm{exc}}^{\mathrm{cap}}=0$. In contrast, at high projectile speeds we assume a constant differential cross section $d \sigma$ cap $/ d E$ in the spectrum of bound projectile states (upper colored areas in Figs. 2(b) and 2(d), at the projectile), leading to a mean projectile binding energy of $I_{\text {proj; } 1}(q-1) / 2$ as indicated in Figs. 2(b) and 2(d). For both cases, we have $\Delta E_{\mathrm{exc}}^{\mathrm{cap}}=I_{\mathrm{tar} ; 1}-0.5 I_{\mathrm{proj} ; 1}(q-1)$. For low speeds and small projectile-ionization potentials (in comparison to the target), resonant capture is impossible and we assume that capture leads exclusively to ground-state population with $\Delta E_{\mathrm{exc}}^{\mathrm{cap}}=I_{\mathrm{tar} ; 1}-I_{\text {proj; } 1}(q-1)$ [see Fig. 2(c)]. The above four cases (dependent on speed and ionization potential) are the asymptotic results of Eq. (2), where the Figs. 2(a) and 2(b) correspond to the upper condition of Eq. (2) and Figs. 2(c) and 2(d) correspond to the lower formula in Eq. (2). For consideration of the velocity dependence, we have used simple switching functions dependent on $v_{p}^{2} / 2$. These switching functions are expected to lead to uncertainties of up to about $10 \%$ at finite speeds (as long as target inner shells do not contribute).

As a next step, we make use of the classical rate equations [37] that determine the projectile charge-state distribution. We may consider charge-state equilibrium [related to the steady-state conditions $f(q, t)=$ constfor all values of $q$ ] and neglect multielectron processes ( $n$-electron capture or loss). Neglecting excitations (in a first step) and summing now over all charge states above a certain fixed value of $q$, we find for all such values of $q$ (with $0 \leqq q<Z$ ),

$$
f(q) \sigma^{\text {loss }}(q)=f(q+1) \sigma^{\mathrm{cap}}(q+1),
$$

as follows directly from probability conservation within the charge-state range. At this point, a short discussion about the effect of solid/gas differences for Eq. (3) might be appropriate. Of course, the fits to experimental charge-state distributions [41] depend on the state of the target system. The target atomic density governs the mean free path between subsequent collisions and charge equilibration depends on the ratio of the collision frequency and the photon decay rates (if we neglect Auger transitions). In the case of low speeds or low target densities (gas targets), excited projectile states decay before the next collision process takes place. Thus, bound-state excitation of projectile electrons plays no role and $\sigma^{\text {cap }}(q)$ accounts for capture into all projectile shells (total capture cross section). On the contrary, at high collision frequencies, there is a fraction of populated excited states accumulated from previous collisions due to projectile-bound-state excitation and capture into excited states. These facts have an influence on the distribution of electronic projectile states (increased number of excitations) and on the charge-state fractions $f(q)$, governed by the increased effective electron-loss cross sections (the capture cross sections remain nearly unaffected). Without the (minor) influence from Auger transitions, however, Eq. (3) still remains valid (when the loss cross section accounts for the actual state distribution). Now, reindexing Eq. (1) and inserting Eq. (3) into the resulting sum yields

$$
\begin{aligned}
S_{e}^{\mathrm{cap}}(q) & =\sum_{q=0}^{Z_{p}-1} f(q+1) \sigma^{\mathrm{cap}}(q+1) \Delta E^{\mathrm{cap}}(q+1) \\
& =\sum_{q=0}^{Z_{p}-1} \sum_{\text {state }} f(q) \sigma_{\text {state }}^{\text {loss }}(q) \Delta E^{\mathrm{cap}}(q+1)
\end{aligned}
$$

Now one might estimate the electron-loss cross section $\sigma^{\text {loss }}(q)$ (for all deeply bound projectile shells, but without projectile excitations) from the corresponding gross electronic stopping cross $\operatorname{section} S_{e, \operatorname{proj}}(q)$ related to projectile excitation and ionization (in the projectile system) for each charge state $q$ and for each populated bound state $i$. This relation is extracted from a fit to the theoretical scaled cross-section ratio

$$
R_{i}^{\text {loss }}(q)=I_{\mathrm{proj} ; i}(q) \sigma_{i}^{\text {loss }}(q) / S_{e, \text { proj } ; i}(q)
$$

as shown in Fig. 3. The quantities $\sigma_{i}^{\text {loss }}(q)$ and also $S_{e, \text { proj } ; i}(q)$ that determine the ratio in Fig. 3 are obtained from highly accurate numerical benchmark calculations using colossal basis sets (700-1000 projectile-centered states) in AOCC calculations [8-11]. These time consuming calculations (numerical solutions of the Schrödinger equation for independent electrons) have been performed for four different collision systems, i.e., for (static) neutral $\mathrm{H}$ and $\mathrm{He}$ targets interacting with individual $\mathrm{H} 1 s, \mathrm{He} 1 s$, and $\mathrm{C} 2 s$ electrons of neutral $\mathrm{H}, \mathrm{He}$, and $\mathrm{C}$ projectiles. The resulting precision correction factors (numerical uncertainties are expected to be about $2 \%$ ) are given as a function of a reduced energy $E_{\text {red }}$, computed from the projectile velocity, from the binding energy $I_{\text {proj }}(q)$, and the mean of the kinetic electron energy 


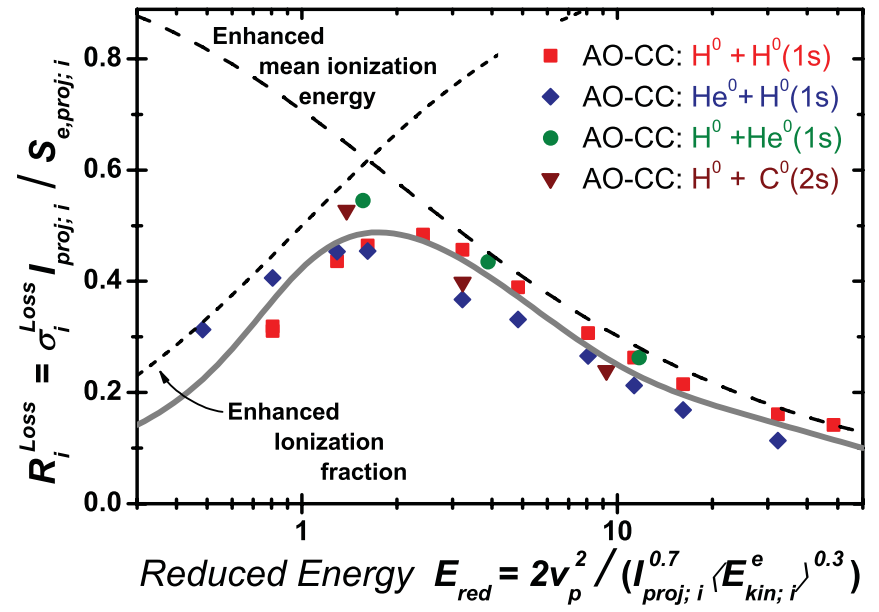

FIG. 3. (Color online) Scaled cross-section ratio for projectile energy losses as discussed in the text (evaluated for neutral $\mathrm{H}$ and $\mathrm{He}$ target potentials interacting with $\mathrm{H} 1 s, \mathrm{He} 1 s$, and $\mathrm{C} 2 s$ electrons of neutral $\mathrm{H}, \mathrm{He}$, and $\mathrm{C}$ projectiles).

$E_{\text {kin }}^{e}(q)$ of the projectile shell under consideration. This choice of a reduced energy accounts for electronic screening effects and maximizes the overlap between computed curves for different initial bound states. Note that these data are directly related to the so-called $W$ value [52-54] of ionizing events (the average energy to produce an ion pair in a gas of low atomic density).

The four computed AOCC ratios show a comparable behavior, namely, a rising curve at low velocities that reaches a maximum ratio of about 0.5 at a reduced energy between 1 and 3. At larger velocities all curves are falling again. Considering that the projectile-related stopping cross section $S_{e, \text { proj }}(q)$ may be expressed as a product of mean excitation energy $\left\langle\Delta E_{\text {proj }}(q)\right\rangle$ and an effective cross section $\sigma_{\text {proj }}^{\text {effective }}(q)$, this tendency is easily explained. At low velocities projectile inelastic processes are dominated by excitation. Thus, we have $\left\langle\Delta E_{\text {proj }}(q)\right\rangle \approx I_{\text {proj; } 1}(q)$ for low velocities (excitation energies are close to the first ionization potential) and the effective inelastic projectile cross section is roughly $\sigma_{\text {proj }}^{\text {effective }}(q)=2 \sigma^{\text {loss }}(q)$, when the maximum electronic energy transfer is equal to the projectile binding energy $\left(E_{\mathrm{red}}=\right.$ 1 in Fig. 3). At this velocity, projectile excitation is about equal to projectile ionization. A modified linear dependence, with the above cross-section condition and a restriction to unity, is shown by the short-dashed curve in Fig. 3. At high velocities, the energy losses are dominated by ionization, corresponding to $\sigma_{\text {proj }}^{\text {effective }}(q) \approx \sigma^{\text {loss }}(q)$. In this energy regime, however, the mean excitation energy for inelastic processes may be approximated by a restricted geometrical average between binding energy and maximum electronic energy

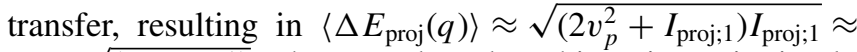
$I_{\text {proj; } 1} \sqrt{\left(E_{\text {red }}+1\right)}$. The curve based on this estimate is simply $y=\left(E_{\text {red }}+1\right)^{-0.5}$ and it is shown as a long-dashed curve in Fig. 3. The product of both dashed curves (accounting for variations of the mean energy transfer as well as of the effective cross section) is not far from our numerical results, indicating that we understand the functional dependence at least qualitatively.

The gray solid curve in Fig. 3 is a fit to the numerical AOCC results for $R_{i}^{\text {loss }}(q)$ and it is used below to obtain a reasonable estimate for the projectile-ionization (pure electron-loss) cross section. The uncertainty of this fit curve is assumed to exceed $15 \%$ (probably due to quasimolecular orbital effects, which are specific for each collision system), especially at low velocities. From Eqs. (4) and (5), we derive

$$
\begin{aligned}
S_{e}^{\mathrm{cap}}= & \sum_{q=0}^{Z_{p}-1} \sum_{i} f(q) \Delta E^{\mathrm{cap}}(q+1) R_{i}^{\mathrm{loss}}(q) \\
& \times S_{e, \operatorname{proj} ; i}(q) / I_{\mathrm{proj} ; i}(q),
\end{aligned}
$$

together with Eq. (2). The above treatment reminds one of Sigmund's ICRU contribution [5], where the computation of an equilibrium energy loss due to capture is sketched in four

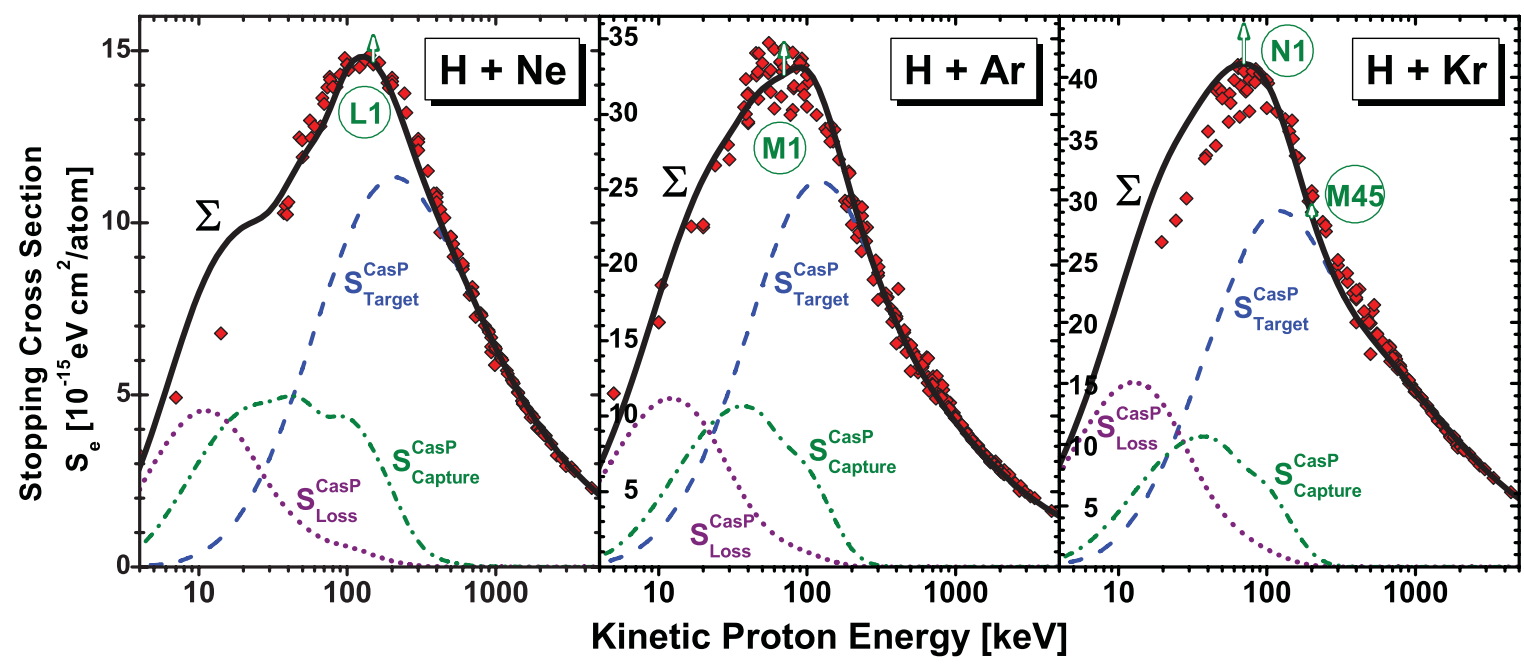

FIG. 4. (Color online) Projectile-energy dependence of the electronic energy loss of charge-equilibrated hydrogen beams in different heavy rare gases $(\mathrm{Ne}, \mathrm{Ar}$, and $\mathrm{Kr}$ ). Experimental values (red diamonds) are taken from the data collection of Paul [55]. Dashed, dotted, and dashed-dotted curves are charge-weighted partial energy-loss cross sections. The solid black curve denoted $\Sigma$ corresponds to the sum of these partial contributions. The open arrows and shell indicators $\left(L_{1}, M_{1}, N_{1}, M_{45}\right)$ are explained in the text. 


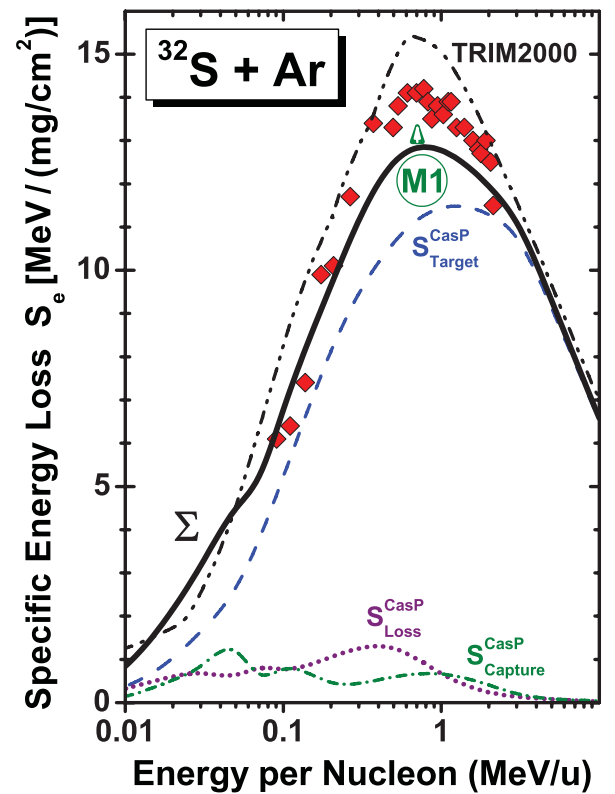

FIG. 5. (Color online) Ab initio electronic energy loss of chargeequilibrated sulfur ions in Ar, similar as in Fig. 4. Experimental values are taken from the data collection of Paul [55]. The dash-dot-dot curve corresponds to the TRIM2000 stopping-power fit [56].

sentences. However, the current more involved treatment regarding cross sections and energy transfers is expected to deviate significantly from such previous estimates. In the following, we apply Eqs. (2) and (6), together with CASP calculations for the target and projectile excitation/ionization processes. The summed stopping cross section is compared with experimental data obtained from the data collection of Paul [55].

\section{RESULTS AND DISCUSSION}

Figure 4 displays UCA results for the partial energy-loss cross sections related to excitation plus ionization of bound projectile electrons (purple dotted curve denoted by "loss"), excitation plus ionization of bound target electrons (blue dashed curve denoted by "target"), and electron transfer from bound target to bound projectile states (these charge-exchange processes are denoted by "capture" and are displayed as an olive-colored dashed-dotted curve). The partial stopping cross-section curves show typical tendencies. At low velocities $S_{e}$ is dominated by projectile electron-loss and projectile excitation processes. At somewhat higher velocities the stopping contribution by electron capture maximizes and involves significant variations of the functional shapes. The arrows and shell indicators $\left(L_{1}, M_{1}, N_{1}, M_{45}\right)$ show upper estimates for the influence of deeper bound target states on the energy transfer in capture events [not accounted for in Eq. (2)]. The target contribution always involves the highest partial energy-loss cross section and determines the asymptotic high-energy behavior almost completely.

The thick solid curve (marked with " $\Sigma$ ") is the total electronic slowing-down cross section $S_{e}$, i.e., the sum of the above three partial stopping cross sections averaged over the projectile charge states. These black curves may directly be compared with the available experimental data (shown in the figure by red diamond symbols) from the web pages by Paul [55]. The comparison shows good agreement between UCA and experimental data at intermediate to high energies. At low kinetic energies (below $20 \mathrm{keV}$ ) the maximum deviation between experiment and UCA reaches $20 \%$ for the $\mathrm{Ne}$ and $\mathrm{Kr}$ targets. For the Ar target, however, we find good overall agreement between experiment and ab initio theory.

Figure 5 displays results and data similar to Fig. 4, but obtained for heavy ions, namely, for S on Ar. It is noted that the capture and loss fractions are relatively small in this heavy-ion case. These fractions reach 5-10\% for S $+\mathrm{Ar}$, in comparison to $30-35 \%$ for the proton results in Fig. 4. The charge-state fractions in Figs. 4 and 5 have been taken directly from the CASP program. Consideration of the Ar- $M_{1}$ shell for the initialstate energy in capture events leads only to a minor increase of the results in Fig. 5. It is seen, however, that there is good agreement between experimental and theoretical energy-loss cross sections. Typical deviations are below $\sim 10 \%$, pointing to accurate nonlinear corrections. Note that the accuracy of our $a b$ initio results is comparable to the electronic energy-loss fit incorporated in the TRIM2000 code [56].

\section{CONCLUSIONS}

In conclusion, in this paper we have successfully included the electron-capture contribution into the CASP energy-loss code. Good agreement with experimental data has been achieved for high to intermediate energies. At energies below $\sim 20 \mathrm{keV}$, however, we find significant deviations between experiment and theory in some cases. Theoretical uncertainties are always expected in this energy regime, due to quasimolecular orbital effects that may influence low-energy charge-state fractions and electron-capture cross sections. Furthermore, derivations of this type (see also Ref. [5]) are sensitive to multielectron transitions [in Eq. (3)] and to the assumed relation between the energy-loss cross section and the total ionization cross section in Eq. (6). The present results also indicate that electron capture is very important for light ions at intermediate speeds and less important for heavy ions.
[1] P. L. Grande and G. Schiwietz, in Advances in Quantum Chemistry, edited by J. Sabin (Elsevier, New York, 2004), Vol. 45, pp. 7-46.

[2] G. Schiwietz et al., J. Phys. B 29, 307 (1996).

[3] G. Schiwietz et al., Nucl. Instrum. Methods Phys. Res. B 226, 683 (2004).
[4] S. A. Cruz, C. Vargas-Aburto, D. K. Brice, E. V. Alonso, and D. G. Armour, Phys. Rev. A 27, 2403 (1983).

[5] P. Sigmund and A. Schinner, Nucl. Instrum. Methods Phys. Res. B 195, 64 (2002); corresponding treatment of electron capture is sketched in ICRU Report 73, J. ICRU 5, 118 (2005) 
[6] J. Bang and J. M. Hansteen, K. Dan. Vidensk. Selsk. Mat. Fys. Medd. 31, 13 (1959).

[7] C. C. Montanari, J. E. Miraglia, and N. R. Arista, Phys. Rev. A 66, 042902 (2002).

[8] G. Schiwietz, Phys. Rev. A 42, 296 (1990).

[9] P. L. Grande and G. Schiwietz, Phys. Rev. A 44, 2984 (1991).

[10] G. Schiwietz and P. L. Grande, Nucl. Instrum. Methods Phys. Res. B 69, 10 (1992).

[11] P. L. Grande and G. Schiwietz, Phys. Rev. A 47, 1119 (1993).

[12] I. Wojciechowski and B. J. Garrison, Surf. Sci. 527, 209 (2003).

[13] A. Duvenbeck, B. Weidtmann, O. Weingart, and A. Wucher, Phys. Rev. B 77, 245444 (2008).

[14] J. J. Dorado and F. Flores, Phys. Rev. A 47, 3062 (1993).

[15] A. Arnau, M. Penalba, P. M. Echenique, F. Flores, and R. H. Ritchie, Phys. Rev. Lett. 65, 1024 (1990).

[16] A. F. Lifschitz and N. R. Arista, Phys. Rev. A 57, 200 (1998).

[17] A. F. Lifschitz and N. R. Arista, Phys. Rev. A 69, 012902 (2004).

[18] P. L. Grande and G. Schiwietz, J. Phys. B 28, 425 (1995).

[19] C. O. Reinhold and J. Burgdörfer, J. Phys. B 26, 3101 (1993).

[20] P. Sigmund and A. Schinner, Eur. Phys. J. D 12, 425 (2000).

[21] P. Sigmund and A. Schinner, Nucl. Instrum. Methods Phys. Res. B 243, 457 (2006).

[22] P. D. Fainstein, G. H. Olivera, and R. D. Rivarola, Nucl. Instrum. Methods Phys. Res. B 107, 19 (1996).

[23] G. Maynard, G. Zwicknagel, C. Deutsch, and K. Katsonic, Phys. Rev. A 63, 052903 (2001).

[24] A. Dalgarno and G. W. Griffing, Proc. R. Soc. London, Ser. A 232, 423 (1955); D. R. Bates and G. Griffing, Proc. Phys. Soc., London, Sect. A 66, 961 (1953).

[25] T. Kaneko, Phys. Rev. A 33, 1602 (1986).

[26] N. M. Kabachnik, V. N. Kondratev, and O. V. Chumanova, Phys. Status Solidi B 145, 103 (1988).

[27] M. Inokuti, Rev. Mod. Phys. 43, 297 (1971).

[28] P. L. Grande and G. Schiwietz, Nucl. Instrum. Methods Phys. Res. B 267, 859 (2009).

[29] P. L. Grande and G. Schiwietz, Phys. Rev. A 58, 3796 (1998).

[30] P. L. Grande and G. Schiwietz, Nucl. Instrum. Methods Phys. Res. B 195, 55 (2002).

[31] G. Schiwietz and P. L. Grande, Nucl. Instrum. Methods Phys. Res. B 153, 1 (1999).

[32] The most recent casp program version (currently version 5.0) may be downloaded from [http://www.casp-program.org/]. Note that it does not contain the electron-capture estimate discussed in this work.
[33] P. L. Grande and G. Schiwietz, Nucl. Instrum. Methods Phys. Res. B 136-138, 125 (1998).

[34] F. Herman and S. Skillmann, Atomic Structure Calculations (Prentice-Hall, Englewood Cliffs, NJ, 1963).

[35] J. D. Jackson, Classical Electrodynamics (Walter de Gruyter, Berlin, 1981).

[36] G. M. de Azevedo, P. L. Grande, and G. Schiwietz, Nucl. Instrum. Methods Phys. Res. B 164, 203 (2000)

[37] H.-D. Betz, Rev. Mod. Phys. 44, 465 (1972).

[38] J. P. Rozet, A. Chetioui, P. Bouisset, D. Vernhet, K. Wohrer, A. Touati, C. Stephan, and J. P. Grandin, Phys. Rev. Lett. 58, 337 (1987).

[39] G. Schiwietz, Radiat. Eff. Defects Solids 112, 195 (1990).

[40] J. P. Rozet et al., Phys. Lett. A 274, 37 (2000).

[41] G. Schiwietz and P. L. Grande, Nucl. Instrum. Methods Phys. Res. B 175-177, 125 (2001); G. Schiwietz, M. Roth, K. Czerski, F. Staufenbiel, and P. L. Grande, ibid. 226, 683 (2004).

[42] W. H. Barkas, N. J. Dyer, and H. H. Heckmann, Phys. Rev. Lett. 11, 26 (1963).

[43] G. de M. Azevedo, P. L. Grande, M. Behar, J. F. Dias, and G. Schiwietz, Phys. Rev. Lett. 86, 1482 (2001); L. L. Araujo, P. L. Grande, M. Behar, J. F. Dias, A. F. Lifschitz, N. R. Arista, and G. Schiwietz, Phys. Rev. A 70, 032903 (2004).

[44] G. Schiwietz and P. L. Grande, Nucl. Instrum. Methods Phys. Res. B (in press), doi: 10.1016/j.nimb.2011.07.023.

[45] P. Sigmund, Phys. Rev. A 26, 2497 (1982).

[46] P. Sigmund and L. G. Glazov, Eur. Phys. J. D 23, 211 (2003).

[47] D. P. Dewangan and J. Eichler, J. Phys. B 19, 2939 (1986).

[48] D. Belkic, R. Gayet, J. Hanssen, and A. Salin, J. Phys. B 19, 2945 (1986).

[49] J. Eichler, Phys. Rev. A 32, 112 (1985).

[50] H. Ryufuku, K. Sasaki, and T. Watanabe, Phys. Rev. A 21, 745 (1980).

[51] A. Niehaus, J. Phys. B 19, 2925 (1986).

[52] J. A. Phipps, J. W. Boring, and R. A. Lowry, Phys. Rev. 135, A36 (1964).

[53] F. P. Santos, T. H. V. T. Dias, P. J. B. M. Rachinhas, C. A. N. Conde, and A. D. Stauffer, J. Appl. Phys. 89, 8202 (2001).

[54] M. Inokuti and E. Eggarter, J. Chem. Phys. 86, 3870 (1987).

[55] Extensive experimental energy-loss tabulations are maintained by $\mathrm{H}$. Paul at [http://www.exphys.jku.at/stopping/].

[56] Current versions of the code by F. Ziegler, and J. P. Biersack may be obtained from http://www.SRIM.org. 